Review article

\title{
A short review on leptospirosis: Clinical manifestations, diagnosis and treatment
}

\author{
Chinju Susan Chacko ${ }^{a}$, Shravya Lakshmi S ${ }^{b}$, Anjali Jayakumar ${ }^{c}$, Steffy Ligi Binu ${ }^{c}$, \\ Ramesh Datta Pant ${ }^{\mathrm{d}}$, Ashish Giri ${ }^{\mathrm{e}}$, Sharad Chand ${ }^{\mathrm{c}, * *}$, Nandakumar UP ${ }^{\mathrm{c}, *}$ \\ ${ }^{a}$ Department of Pharmacy Practice, College of Pharmaceutical Sciences, Dayananda Sagar University, Bangalore, India \\ ${ }^{\mathrm{b}}$ Department of Pharmaceutics, College of Pharmaceutical Sciences, Dayananda Sagar University, Bangalore, India \\ ${ }^{\mathrm{c}}$ Nitte (Deemed to be University), Nitte Gulabi Shetty Memorial Institute of Pharmaceutical Sciences (NGSMIPS), Department of Pharmacy Practice, Mangaluru, \\ Karnataka, 575018, India \\ ${ }^{\mathrm{d}}$ Covance India Pharmaceutical Services Private Limited, Bengaluru, Karnataka, India \\ e Department of Pharmacy Practice, TVM College of Pharmacy, Ballari, Karnataka, 583103, India
}

\section{A R T I C L E I N F O}

\section{Keywords:}

Leptospirosis

Pulmonary hemorrhage

Renal failure

MAT test

Penicillin G

\begin{abstract}
A B S T R A C T
In the last few decades, leptospirosis has emerged globally as a fatal infectious disease. Yet, an understanding of the pathogenesis of leptospirosis continues to remain within the grey areas of scientific evidence. In a majority of the cases, the infections are asymptomatic. The benchmark test for leptospirosis is the microscopic agglutination test, commonly known as the MAT test. The standard of treatment is oral doxycycline, although penicillin and azithromycin can also be used. In this review, we aim to elaborately discuss the symptoms, diagnosis and the approved therapeutic regimen of leptospirosis.
\end{abstract}

\section{Introduction}

The causative agent for leptospirosis is the bacteria belonging to leptospira spps. ${ }^{1}$ The incidence of leptospirosis is increasing on a global scale and developing countries are no exception to this. ${ }^{2}$ This rising statistic can be attributed to the lack of accessibility to appropriate diagnostic procedures. ${ }^{1}$ Transmission and clinical presentations of leptospirosis seem to differ in different environmental and socioeconomic situations. ${ }^{3}$ According to the review of scientific literature, the primary pool of cases comprises young men. ${ }^{4}$ In some cases, it has been observed that the actual rate is higher than the estimated rate. This is because many often go undiagnosed due to the existing poor diagnostic testing facilities and the non-specific nature of the disease. ${ }^{5}$ Pulmonary hemorrhage, renal failure and jaundice are predominantly found in urban areas. $^{3}$ In recent years, it has also surfaced as the disease of adventure travelers affecting individuals who actively participate in water sports. ${ }^{6}$ However, the medical literature has concluded leptospirosis to be a treatable disease and is usually reported in low-income countries of tropical regions. ${ }^{7}$ The incidence of disease occurrence is ten times higher in these areas due to a combination of factors such as high temperature, humidity, rainfall and socioeconomic causes such as poor sanitation and close contact with domestic animals, all of which favors the growth of the bacteria. ${ }^{5}$ In developed countries like the United States of America, leptospirosis has been regarded as a neglected disease. $^{8}$

Travel related leptospirosis is considered as a significant source of infection in developed countries. As per the results of a study conducted in the United Kingdom, nearly half of the reported cases were found to have a travel history to tropical regions. ${ }^{5}$ In India; only less than 10,000 cases are reported every year, although the estimated numbers of cases lie between 0.1 and 1 million. The bulk of the cases are usually reported from the states of Gujarat, Maharashtra, Tamil Nadu and Kerala. ${ }^{1,2}$ Infections typically result from both direct and indirect exposure or contact with the urine of infected animals and are said to also spread through water or soil. ${ }^{9}$ The lack of active surveillance, awareness and a complicated clinical diagnosis is the established reasons for the rising trends in the disease. ${ }^{8}$ In recent years, exposure to recreational water has also begun to be reviewed as a significant risk factor that contributes to

\footnotetext{
* Corresponding author. Nitte (Deemed to be University), Nitte Gulabi Shetty Memorial Institute of Pharmaceutical Sciences (NGSMIPS), Department of Pharmacy Practice, Mangaluru, Karnataka, 575018, India.

*: Corresponding author. Nitte (Deemed to be University), Nitte Gulabi Shetty Memorial Institute of Pharmaceutical Sciences (NGSMIPS), Department of Pharmacy Practice, Mangaluru, Karnataka, 575018, India.

E-mail addresses: sureechand193@gmail.com (S. Chand), nandakumarvtkv@gmail.com (N. UP).
} 
the spread of leptospirosis. ${ }^{7}$ The organism may enter the human body via abrasions or cuts in the mucous membrane, while the chances are very slim for the causative organism to penetrate through intact skin. Pathogenic leptospira is very rarely seen to transmit the disease via the respiratory or the fecal-oral route. ${ }^{6,7}$ Once the organism enters a human body, it multiplies in the bloodstream, leading to its homogeneous dissemination throughout the body. The pathogen affects nearly all organ systems because the spirochetes have the capability to cross tissue barriers before the antibodies of the host get an opportunity to eliminate them from the bloodstream. ${ }^{5}$ The under-reporting of leptospirosis can mainly be attributed to clinical misdiagnosis as the manifestations closely resemble the symptoms of other diseases such as malaria, dengue fever, rickettsia disease, yellow fever and HIV infection. ${ }^{7}$

Pulmonary hemorrhage, Weil syndrome with jaundice and acute kidney injury (AKI) are seen in only $10 \%$ of the affected individuals. ${ }^{10}$ Weil syndrome occurs only in severe forms of leptospirosis and is denoted by a failure of the renal system accompanied by hepatomegaly, liver impairment and/or an alteration in the levels of consciousness. The syndrome usually occurs among those who have stated a history of exposure to infected animals. ${ }^{11}$ Leptospirosis induced acute kidney injury is often characterized by hypokalemia. ${ }^{10}$ Besides, in some cases, severe forms of leptospirosis can be life-threatening as it results in multiple organ system failures, including hepatic and vascular damage, pulmonary hemorrhage, renal failure and the observance of characteristic muscle lesions. ${ }^{12}$ There are several factors that are interrelated in AKI associated with leptospirosis and it is characterized by rhabdomyolysis, hyperbilirubinemia, hypovolemia and the direct nephrotoxic action of leptospira. ${ }^{4}$ Experimental and clinical studies indicate alterations in the tubular functions, which further causes changes in the proximal tubule and the glomerular filtration rate. ${ }^{12}$ The major histological findings noted in the patients with leptospirosis are interstitial nephritis and acute tubular necrosis. ${ }^{4}$ The studies conducted among humans and animals illustrated a down regulation in the expression of proximal sodium and tubular water transporter, aquaporin one, and a higher renal expression of the Na-k-2cl co-transporter NkccQ. ${ }^{12}$ Acute kidney injury associated with leptospirosis is usually non-oliguric in nature. The decline in the glomerular filtration rate caused by alterations to tubular function forms the basis for the repeated occurrence of hypokalemia during advanced stages of the disease. ${ }^{4}$ Acute kidney injury and the increased mortality rate associated with it can be attributed to acute respiratory distress syndrome, pulmonary edema, alveolar hemorrhage, or sometimes a combination of all of these. ${ }^{10}$ Mortality due to leptospirosis induced acute kidney injury is around $20 \%{ }^{4}$

\section{Clinical presentation}

The clinical symptoms of leptospirosis are highly variable and nonspecific. Majority of the cases remain subclinical or asymptomatic and while symptoms are present, it typically manifests 2-30 days after the initial exposure. ${ }^{5}$ The infection is responsible for various clinical features ranging from subclinical symptoms to fatal pulmonary hemorrhage and Weil's syndrome, which makes the therapy challenging for the treating clinicians. ${ }^{1,13}$ Majority of the patients present with fever at the time of hospital admission. ${ }^{14}$ Symptoms include the abrupt onset of fever with chills, headache and myalgia. ${ }^{5}$ Clinical presentation is often characterized by pyrexia of unknown origin, which causes difficulties in documenting an accurate early diagnosis. ${ }^{8}$ Throbbing muscle aches primarily focused on the lower back and calves is a classic symptom. ${ }^{5}$ Ocular findings such as hemorrhages in the subconjunctival region and icterus are also observed. ${ }^{13}$ Other symptoms seen in leptospirosis are associated with the gastrointestinal system, including nausea, vomiting, abdominal pain and diarrhea (Lane AB et al., 2016). Among non-oliguric renal failure patients, gastrointestinal symptoms may contribute to dehydration. ${ }^{15}$ Conjunctival irritation, dry cough, abdominal pain, loose stools, vomiting, meningeal syndrome, cardiac arrhythmia, anuria or oliguria, and skin rash are other reported manifestations. Severe leptospirosis usually leads to multiple organ dysfunctions with the liver, lungs, kidney, and brain being the target organs of damage. ${ }^{13}$ Severe forms of the disease can result in neuro-leptospirosis, hemorrhagic pneumonitis and acute respiratory distress syndrome. ${ }^{8}$ Weil's disease, which is a combination of jaundice and renal failure, is the most clinically recognizable form of leptospirosis. ${ }^{13}$ High conjugated bilirubin levels, cholestatic pain and mild elevations in serum aminotransferase levels are commonly reported among patients with liver impairments. ${ }^{5}$

Pulmonary symptoms include hemorrhagic alveolar syndrome and pulmonary edema, which finally culminates in the occurrence of acute respiratory distress syndrome. ${ }^{5}$ The evidence clearly illustrates that organ dysfunction occurs in the advanced stages of infection. ${ }^{13}$ The frequency of jaundice varies depending upon the virulence of the causative organism. ${ }^{15}$ Non-specific cardiogram abnormalities are also seen if the leptospira severely affects the heart. ${ }^{5}$ The cardiac manifestations include myocarditis, pericarditis, heart block and arrhythmia. ${ }^{15}$ Hematological tests in some patients have confirmed the presence of mild leukocytosis as well as thrombocytopenia. If the disease severely affects the renal system, elevations in the serum creatinine, hypokalemia and hyponatremia are observed. Urine analysis has also confirmed pyuria, proteinuria and the incidence of occasional microscopic hematuria. In some cases, serum amylase and creatine kinase have also been noted to be elevated. ${ }^{5}$ Meningoencephalitis is an indicator of severe leptospirosis. Other neurologic complications include transverse myelitis, hemiplegia and Guillain-Barré syndrome. ${ }^{15}$ Cerebrospinal fluid analysis is mostly consistent with aseptic meningitis, with moderately elevated protein levels, lymphocytic pleocytosis, and normal glucose level. ${ }^{5}$

\section{Diagnostic procedures}

A detailed history of exposure and symptoms plays a prominent role in the confirmation of leptospirosis as its clinical manifestations closely resemble the representations of a variety of other febrile illnesses. ${ }^{5}$ Detection of leptospirosis is achieved by confirming the organism's presence or its metabolites in the body fluids or tissue, using serological tests and the culture method. ${ }^{15}$ It is observed that the antibodies for leptospirosis develop only $3-10$ days after the initial symptoms. Negative serologic test results obtained from the samples that are collected in the first week of illness cannot be used to rule out the presence of infection. Hence, the serology test should be repeated on a sample collected 7-14 days after the primary examination. ${ }^{8}$ Stains of spirochetes are poorly visualized with traditional staining methods and the darkfield microscopy technique is employed for clear visualization. During the first ten days of illness, CSF and blood cultures are extensively useful. ${ }^{5}$ Two types of staining procedures are commonly utilized, namely, histochemical and immunostaining methods. In the histochemical staining procedure, the Warthin-Starry stain is widely using. ${ }^{16}$ Urine cultures of leptospira are expected to be positive even after the second week of illness and may remain positive for another 30 days, even after the resolution of symptoms due to the impact of the organism on the renal tubules. The standard gold test for leptospirosis is the microscopic agglutination test, commonly known as the MAT test. ${ }^{5}$ Other supportive diagnostic tests include ELISA IgM, Immuno DOT and Lateral flow tests. ${ }^{8}$ In some cases, Polymerase chain reaction (PCR) and pathology tests are also employed to confirm the presence of the pathogen. ${ }^{16}$ The following are the guidelines offered in India for diagnosing leptospirosis.

- Faine's criteria- World Health Organization (WHO) guidelines 1982 - Modified Faine's criteria 2004

- Modified Faine's criteria with amendment 2012

- Guidelines for prevention and control of leptospirosis: National Institute of Communicable Diseases (Zoonosis Division). ${ }^{2}$

- Guidelines by the Regional Medical Research Centre (ICMR) and WHO regional office for South-East Asia 
Although there are procedures available for the diagnosis of leptospirosis, the initial assumption may confound with the confirmatory diagnosis. The differential diagnosis of leptospirosis is difficult due to the overlap of its clinical presentations with dengue and dengue hemorrhagic fever, influenza, malaria, enteric fever, toxoplasmosis, hepatitis etc. Hence, the MAT test is preferred to avoid the misdiagnosis of this tropical infection. ${ }^{17}$

\section{Treatment}

Majority of leptospirosis cases are mild and resolve spontaneously. ${ }^{15}$ Minor cases of leptospirosis resolve with time and oral antibiotics, including doxycycline, azithromycin, ampicillin and amoxicillin, are the drugs that are given depending on the severity of the infection. ${ }^{13}$ Initial treatment options are chosen depending on the severity of the illness at presentation. Azithromycin or doxycyclines are the drugs of choice as per standardized guidelines in geographical locations where rickettsial diseases are endemic. ${ }^{5}$ Among the pregnant population and young children, the use of doxycycline is contraindicated. ${ }^{13}$ In some patients, the disease's progression to more severe forms can be prevented by the early initiation of antimicrobial treatment. ${ }^{15}$ Regardless of the duration of symptoms, treatment with antibiotics must be initiated as soon as a provisional diagnosis of leptospirosis is suspected. It is unnecessary to withhold treatment until confirmed lab reports or diagnosis is attained before commencing therapy as a clinical profile and environmental history are of more value. ${ }^{2}$ In severe cases, intravenous penicillin $\mathrm{G}$ has proven to be equally effective as cefotaxime and ceftriaxone. ${ }^{13}$ Early start-up of antibiotics is vital to avoid complications, but the diagnosis must be confirmed by the second week of illness. ${ }^{2}$ Due to antibiotic resistance seen in the pathogens, several studies show no clinical effect with penicillin G. Hence; there has been an interest in assessing the therapeutic efficacy of other antibiotics. Ceftriaxone has proven to be non-inferior to penicillin $\mathrm{G}$ to manage severe cases of leptospirosis. ${ }^{5}$

Treatment for patients with leptospirosis usually involves ampicillin at a dose of $0.5-1 \mathrm{~g}$ IV q6h, intravenous penicillin 1.5 million units IV four times a day, ceftriaxone $1 \mathrm{~g}$ IV once a day or cefotaxime $1 \mathrm{~g}$ IV QID for seven days along with empirical therapy as recommended by WHO. Mild cases are usually treated with either doxycycline $100 \mathrm{mg}$ oral twice a day or amoxicillin $500 \mathrm{mg}$ QID or ampicillin 500-750 mg QID for 7-10 days or azithromycin $500 \mathrm{mg}$ once daily for three days. ${ }^{2}$ Administration of fluids is recommended to correct hypovolemia, hypotension, or bleeding and transfusion with saline/blood is mostly suggested. In patients suffering from complications like acute kidney injury, treatment with fluids or diuretics is initiated in mild cases and dialysis is performed in severe stages of the disease. It is mandatory to facilitate ventilator support for patients who encounter complications like acute respiratory distress syndrome (ARDS) and pneumonia. ${ }^{1,2}$ The dosage of drugs should be adjusted according to the bodyweight of the patient. In the case of pregnant women and children, azithromycin or amoxicillin are the drugs of choice instead of doxycycline. ${ }^{15}$ Chemoprophylaxis is advocated for those that are at a high risk of exposure and hence, a prophylactic oral dose of $200 \mathrm{mg}$ of doxycycline should be administered once a week throughout the period of exposure. ${ }^{2}$

\section{Prevention}

Spreading awareness regarding the routes of transmission of the disease is the ideal path to eradicate leptospirosis. ${ }^{15}$ The most important non-pharmacological preventive measure is to avoid the use of contaminated water. People should also be advised not to swim in water bodies that might possibly be contaminated with the urine of infected animals. Unnecessary exposure to pets and contact with possibly infected animals should be avoided. Outdoor vacations and trips to endemic areas should be avoided as much as possible. The use of protective boots and clothing while coming in contact with stagnant water can reduce one's susceptibility to the infection. Maintaining personal hygiene and frequent hand washing after working in the fields and wet areas is also capable of diminishing the transmission of the disease. ${ }^{18}$ As part of the pharmacological options for prevention, a double-blinded randomized study done in the United States among 940 soldiers revealed that one patient in the oral doxycycline prophylaxis group and twenty patients in the placebo group developed leptospirosis. ${ }^{13}$ A study conducted by Sehgal et al., in 2000 reported that treatment with doxycycline lowered the incidence of the symptomatic disease despite the heavy rainfall amongst the local residents within the endemic areas. ${ }^{15}$ However, the results of Cochrane reviews were not supportive of chemoprophylaxis. ${ }^{13}$ The immunization of domestic pets and agricultural animals can also serve as a crucial step to alleviate the risk of human transmission. ${ }^{15}$

\section{Conclusion}

Leptospirosis is a treatable disease commonly seen in low income and developing nations. The clinical manifestations and the severity of the disease are highly variable. Efficient diagnostic tests and clinical laboratory parameters have been established to confirm the presence of the disease. Available treatment modalities for the vector-borne tropical disease involves the use of antibiotics such as ampicillin, doxycycline, the dosing of which depends on the prognosis of the patient and the progress of the disease.

\section{Funding support}

Nil.

\section{Declaration of competing interest}

Authors declare no conflict of interest.

\section{Acknowledgment}

We authors would like to extend our sincere thanks to the faculty of College of Pharmaceutical Sciences, Dayananda Sagar University and NGSM Institute of Pharmaceutical Sciences, Nitte (Deemed to be University) for their constant support and encouragement during the work.

\section{References}

1 Agrawal SK, Chaudhry R, Gupta N, Arif N, Bhadur T. Decreasing trend of seroprevalence of leptospirosis at all India Institute of medical Sciences New Delhi: 2014-2018. J Fam Med Prim Care. 2018;7(6):1425-1428.

2 Kumar SS. Indian guidelines for the diagnosis and management of human leptospirosis. Medicine Update. 2013:24-29.

3 Johnson MA, Smith H, Joseph P, et al. Environmental exposure and leptospirosis, Peru. Emerg Infect Dis. 2004;10(6):1016-1022.

4 Daher ED, Abreu KL, Silva Junior GB. Leptospirosis - associated acute kidney injury. Brazilian Journal of Nephrology. 2010;32(4):408-415.

5 Lane AB, Dore MM. Leptospirosis: a clinical review of evidence based diagnosis, treatment and prevention. World J Clin Infect Dis. 2016;6(4):61-66.

6 Bharti AR, Nally JE, Ricaldi JN, et al. Leptospirosis: a zoonotic disease of global importance. Lancet Infect Dis. 2003;3(12):757-771.

7 Bandara M, Ananda M, Wickramage K, Berger E, Agampodi S. Globalization of leptospirosis through travel and migration. Glob Health. 2014;10(1):61.

8 Sethi S, Sharma N, Kakkar N, et al. Increasing trends of leptospirosis in northern India: a clinico-epidemiological study. PLoS Neglected Trop Dis. 2010;4(1):e579. https://doi.org/10.1371/journal.pntd.0000579.

9 Trevejo RT, Rigau-Pérez JG, Ashford DA, et al. Epidemic leptospirosis associated with pulmonary hemorrhage-Nicaragua. J Infect Dis. 1995;178(5):1457-1463, 1998.

10 Seguro AC, Andrade L. Pathophysiology of leptospirosis. Shock. 2013;39:17-23.

11 Togal T, Sener A, Yucel N, et al. Intensive care of a Weil's disease with multiorgan failure. J Clin Med Res. 2010;2(3):145-149.

12 De Brito T, Silva AM, Abreu PA. Pathology and Pathogenesis of Human Leptospirosis: A Commented Review. vol. 60. Revista do Instituto de Medicina Tropical de Sao Paulo; 2018. e23.

13 Khan SJ, khattak MB, khan A. Leptospirosis: a disease with global prevalence. J Microbiol Exp. 2018;6(5):219-221.

14 Holla R, Darshan B, Pandey L, et al. Leptospirosis in coastal South India: a facility based study. BioMed Res Int. 2018, 1759125. https://doi.org/10.1155/2018/ 1759125. 
15 Haake DA, Levett PN. Leptospirosis in humans. In leptospira and leptospirosis 2015 springer, berlin, heidelberg. Curr Top Microbiol Immunol. 2015;387:65-97. https:// doi.org/10.1007/978-3-662-45059-8_5.

16 Budihal SV, Perwez K. Leptospirosis diagnosis: competancy of various laboratory tests. J Clin Diagn Res. 2014;8(1):199-202.
17 Wang S, Stobart Gallagher MA, Dunn N. Leptospirosis. [Updated 2020 Aug 10]. in: StatPearls [Internet]. Treasure Island (FL). StatPearls Publishing; 2021, 31st March 2021 https://www.ncbi.nlm.nih.gov/books/NBK441858/.

18 Centre for disease prevention and control [Accessed on 31st March 2021] https:// www.cdc.gov/leptospirosis/features/outdoor-activities.html. 